\title{
¡†rECI\&NE
}

Revista ITECKNE - Universidad Santo Tomás, Seccional Bucaramanga, Colombia, Vol. 19 № 1 enero - junio de 2022

\section{Revisión literaria de despacho económico ambiental considerando análisis bibliométrico}

\author{
Literary review of economic environmental dispatch \\ considering bibliometric analysis
}

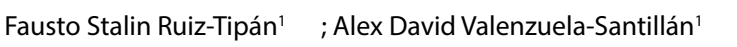

1Universidad Politécnica Salesiana. Quito, Ecuador. fruizt@est.ups.edu.ec, avalenzuela@ups.edu.ec

Fecha de recepción: 05 de junio de 2021. Fecha de aprobación: 19 de agosto de 2021

Resumen- El presente documento realiza un análisis bibliométrico del tema "Environmental Economic Dispatch" para conocer la evolución y las características de su producción científica. Se analizan 736 documentos publicados entre 2000 y 2020 y de estos se extrae los 15 más relevantes, estos serán analizados a detalle tomando en consideración indicadores como el año de publicación, el tema, revista de indexación, cantidad de citas, la temática que aborda y la metodología propuesta. Toda la información se obtuvo de la base de datos Web Of Science (WOS), se empleó esta debido a su alto factor de impacto. En la metodología se expone en análisis bibliométrico que se realizó en el software Vosviewer, el tipo de análisis que se empleo es el de citación y las unidades de análisis trabajadas son las de documentos, fuentes, autores, organizaciones y países. Las unidades de análisis poseen métricas y tablas que ayuden al lector a recopilar la información mostrada de mejor manera, tomando en consideración la cantidad de citas y la cantidad de documentos. En la revisión literaria, se analiza a profundidad los 15 documentos más relevantes obtenidos en el análisis bibliométrico, además se adjunta una tabla a modo de resumen de la temática y metodología propuesta por estos documentos para que el lector sea capaz de identificar más rápido cada uno de estos. En los resultados se destaca el documento más completo para las temáticas propuestas y el documento más citado. Este artículo será una guía útil para los investigadores.

Palabras clave: Despacho Económico Ambiental; Optimización Multiobjetiva;VOSviewer; Análisis Biblimétrico.
Abstract- This document performs a bibliometric analysis of the topic "Environmental Economic Dispatch" to know the evolution and characteristics of its scientific production. A total of 736 documents published between 2000 and 2020 are analyzed and from these the 15 most relevant ones are extracted, these will be analyzed in detail taking into consideration indicators such as the year of publication, the subject, indexing journal, number of citations, the topic addressed and the proposed methodology. All the information was obtained from the Web of Science (WOS) database, which was used due to its high impact factor. In the methodology, the bibliometric analysis was performed in the Vosviewer software, the type of analysis used is that of citation and the units of analysis used are those of documents, sources, authors, organizations, and countries. The units of analysis have metrics and tables that help the reader to compile the information shown in a better way, taking into consideration the number of citations and the number of documents. In the literature review, the 15 most relevant documents obtained in the bibliometric analysis are analyzed in depth, and a table is attached as a summary of the subject matter and methodology proposed by these documents so that the reader will be able to identify each of them more quickly. The results highlight the most complete document for the proposed topics and the most cited document. This article will be a useful guide for researchers.

Keywords: Environmental Economic Dispatch; Multiobjective Optimization;VOSviewer; Bibliometric Analysis.

Citar este artículo como: F. Ruiz Tipán y A. Valenzuela Santillán, Revisión Literaria de Despacho Económico Ambiental considerando análisis bibliométrico, ITECKNE, 19, (1), 2022, pp. 26- 38 doi: https://doi.org/10.15332/iteckne.v19i1.2631 


\section{INTRODUCCIÓN}

Uno de los temas de mayor relevancia de la planificación y operación del sistema eléctrico de potencia (SEP) es la solución eficaz del problema de despacho económico (DE). Con el aumento progresivo de la demanda de energía, la escasez de recursos energéticos y el presuroso ascenso en los precios del combustible, asegurar un funcionamiento económico óptimo de las unidades generadoras de energía térmica se ha convertido en una necesidad [1]

La producción de energía eléctrica medida en megavatios de una unidad de generación térmica está relacionada con el costo del combustible de entrada medido en dólares por hora en forma cuadrática. Desde una perspectiva ideal, las características de entrada-salida de una unidad caldera-turbina-generador se caracterizan por una curva suave y convexa. Sin embargo, la inclusión de pérdidas de transmisión de la red, zonas de operación prohibida (ZOP), carga de punto de válvula (CPV), múltiples máquinas de combustible (MMC), entre otros, ocasiona que el problema de DE convexo sea no convexo y no lineal, así, la curva no convexa de una unidad generadora puede comprender múltiples puntos locales [2].

En [3], se expone que las unidades de generación térmicas producen contaminantes ambientales y emisiones atmosféricas como, dióxido de azufre $\left(\mathrm{SO}_{2}\right)$, óxido de nitrógeno $\left(\mathrm{NO}_{x}\right)$ y dióxido de carbono $\left(\mathrm{CO}_{2}\right)$, que dañan la salud de los seres humanos, la fauna y la flora. Por ello, las empresas eléctricas se han visto presionadas para reducir las emisiones de las centrales térmicas, aunque diferentes soluciones como el uso de combustibles con bajas emisiones de carbono, la sustitución de quemadores de combustible anticuados y el uso de equipos de limpieza poscombustión logran algunos niveles de reducción de emisiones, se sabe que el despacho teniendo en cuenta las emisiones es la mejor opción [4]. El despacho económico ambiental (DEA) se consideró como el mejor enfoque para proporcionar la programación óptima de las unidades generadoras para satisfacer la demanda del sistema, siempre que se minimicen las pérdidas de transmisión, el costo de producción y las emisiones y se cumplan todas las demás restricciones [1]. Con este fin, los investigadores han propuesto una serie de metodologías que pueden clasificarse en dos grandes grupos: técnicas de programación matemática y métodos de optimización metaheurísticos.

Dentro de las técnicas de programación matemática se tiene métodos numéricos deterministas como el método de los multiplicadores de Lagrange [5], método de iteración Lambda [6], el método del punto de base y factores de participación [6], el método del punto interior [7], el método de gradiente [8], el método de Newton Raphson [9], la programación lineal [10], la programación no lineal [11], algoritmo de programación cuadrática [12], el método de programación dinámica [13], la técnica de descomposición [14], el enfoque de espacios de decisión ventajosos [15], la aproximación de serie de Maclaurin [16], entre otros, estas técnicas de programación no se pueden aplicar para un problema de DE complicado y no lineal, debido a que a menudo atrapan un mínimo local, además, estas técnicas no se adaptan eficazmente a los problemas de DE que involucran funciones de costo de combustible no lineales o no convexas.

Dentro de los métodos de optimización metaheurísticos se incluyen: la optimización del enjambre de partículas (PSO) [17], algoritmo genético [18], evolución diferencial [19], programación evolutiva [20], optimización basada en biogeografía [21], recocido simulado [22], optimización de colonias de hormigas [23], algoritmos de abejas [24], optimización de la búsqueda de bacterias [25], algoritmo de luciérnaga [26], algoritmo de búsqueda de cuco [27], algoritmo de murciélago [28], optimización de lobo gris [29], algoritmo de salto de rana [30], algoritmo de polinización de flores [31], optimización basada en la enseñanza-aprendizaje [32], algoritmo de búsqueda de tabú [33], algoritmo de búsqueda de armonía [34], algoritmo de competencia imperialista [35], etc. Estas técnicas metaheurísticas tienen el potencial para asegurar una solución global o casi global mientras se manejan curvas de costos discontinuas y no diferenciables.

Para ayudar a los investigadores a desarrollar técnicas para abordar todos los problemas pendientes, este artículo presenta un estudio de las soluciones propuestas al problema de DEA. La estructura de este artículo se presenta en cuatro secciones principales. En la sección 2, se detalla el problema de despacho económico. En la sección 3, se precisa el problema de despacho económico ambiental. La sección 4 , consta de la metodología que se utiliza para determinar los artículos de mayor relevancia. La sección 5, muestra la revisión literaria de los artículos más relevantes que buscan dar solución al problema de DEA. La sección 6 concluye el presente artículo.

\section{DESPACHO ECONÓMICO (DE)}

En [1], se menciona que el problema del DE es principalmente un problema de optimización restringido donde el costo total de combustible de todas las unidades térmicas en línea de una planta de energía debe aminorarse mientras se determina el nivel de producción de energía en cada una de las unidades generadoras durante un período específico. Las restricciones están en forma de igualdades y desigualdades que deben cumplirse al resolver el problema de DE. El problema del despacho restringido se puede apreciar de diferentes formas, se detalla estas en la subsección siguiente.

\subsection{Problema de DE}

En [36] se describe la función objetivo de este problema estándar ignorando los efectos CPV.

$$
\min F_{T}=\sum_{i=1}^{n} F_{i}\left(P_{i}\right)=\sum_{i=1}^{n}\left(a_{i}+b_{i} P_{i}+c_{i} P_{i}^{2}\right)
$$

Donde $F_{T}$ y $F_{i}\left(P_{i}\right)$ denotan el costo total del combustible y el costo de la $i$-ésima unidad generadora en $\$ / \mathrm{h}$ respectivamente. $P_{i}$ representa la $i$-ésima potencia de salida de la unidad generadora en $\mathrm{MW}, n$ denota el número total 
de unidades generadoras; $a_{i}, b_{i}$ y $c_{i}$ son los coeficientes de costo de la -ésima unidad generadora. De (1) se puede determinar que el costo de combustible de cada una de las unidades generadoras es directamente proporcional con la potencia de salida entregada por esta al SEP y tradicionalmente está modelado por una función cuadrática.

\subsection{Problema de DE con efecto de CPV}

En generadores de turbina de vapor de tamaño considerable, debido a la apertura secuencial de varias válvulas de admisión de vapor para satisfacer la demanda progresiva, las características de entrada-salida de una unidad generadora se torna no convexa. Esto resulta en curvas de costos no lineales, no convexas y no suaves. Para incluir los efectos $\mathrm{CPV}$, se incorpora una no linealidad de orden superior en la función de costo cuadrático. Por lo tanto, la función objetivo a minimizar toma siguiente forma.

$$
\begin{aligned}
& \min F_{T}=\sum_{i=1}^{n} F_{i}\left(P_{i}\right), \quad \text { Si } P_{i}, \min \leq P_{i} \leq P_{i}, \max \\
& \min F_{T}=\sum_{i=1}^{n}\left(a_{i}+b_{i} P_{i}+c_{i} P_{i}^{2}+\left|e_{i} * \operatorname{sen}\left(f_{i} *\left(p_{i, \min }-p_{i}\right)\right)\right|\right)
\end{aligned}
$$

Donde $e_{i}$ y $f_{i}$ denotan los coeficientes de costo de la $i$-ésima unidad generadora que reflejan los efectos CPV. La consideración de las ondulaciones en la curva de tasa de calor de las calderas en forma de término sinusoidal rectificado recurrente en la curva cuadrática de costo introduce mínimos múltiples, lo que ocasiona que el problema de DE no sea convexo ni lineal. Mientras se minimiza $F_{T}$, se debe considerar las limitaciones y restricciones operativas para garantizar una solución óptima. El detalle de las restricciones de igualdad y desigualdad se mencionan a continuación.

\subsubsection{Ecuación de balance de potencia activa}

Esta representa una de las limitaciones básicas para el problema de DE. La potencia total generada de todas las unidades térmicas debe ser la suma de la demanda total del sistema y de las pérdidas del sistema de transmisión. La restricción de igualdad en forma de ecuación se muestra a continuación.

$$
\sum_{i=1}^{n}\left(P_{i}\right)-\left(P_{\text {Load }}+P_{\text {Loss }}\right)=\varphi=
$$

Donde $P_{\text {Load }}$ y $P_{\text {Loss }}$ representan la demanda total del sistema y pérdidas de transmisión respectivamente. Con la finalidad de introducir estas pérdidas, generalmente se utiliza una pérdida de matriz $B$. Se emplea la fórmula de Kron [36] que se aprecia a continuación.

$$
P_{\text {Loss }}=\sum_{i=1}^{n} \sum_{j=1}^{n} P_{i} B_{i j} P_{j}+\sum_{i=1}^{n} B_{i 0} P_{i}+B_{00}
$$

Donde $B_{i j}, B_{i 0}$ y $B_{00}$ significan los coeficientes de pérdidas o el $B$ coeficientes. $B_{i 0}$ representa el $i_{j}$-ésimo elemento de la matriz cuadrada del coeficiente de pérdida,
$B_{10}$ el $i$-ésimo elemento de la pérdida vector de coeficiente, y $B_{00}$ la constante del coeficiente de pérdida.

\subsubsection{Límites de capacidad del generador}

Cada una de las unidades generadoras deben operar dentro de sus límites máximos y mínimo, mientras suple la carga. La restricción de desigualdad de los límites de potencia se muestra a continuación.

$$
P_{i}, \min \leq P_{i} \leq P_{i}, \max
$$

Donde $P_{i}$, min y $P_{i}$, max designan la mínima y máxima potencia de salida de la $i$-ésima unidad generadora respectivamente.

\subsubsection{Generador con límite de velocidad de rampa (LVR)}

Las unidades generadoras en línea deben estabilizarse a los valores de nueva generación en poco tiempo cuando haya un cambio en la demanda de carga. Lograr esto específicamente en el caso de unidades generadoras de vapor más grandes considerando los límites es una tarea desafiante. Prácticamente al imponer límites de velocidad de rampa ascendente y descendente durante cada período de programación, se puede restringir el rango de operación de todas las unidades térmicas comprometidas. Por tanto, el funcionamiento de las unidades está restringido entre los dos periodos de funcionamiento adyacentes. La restricción de desigualdad perteneciente a los límites de la tasa de rampa del generador se caracteriza a continuación:

$P_{i}-P_{i}^{0} \leq U R_{i}$, cuando la generación aumenta

\section{$P_{i}^{0}-P_{i} \leq D R_{i}$ cuando la generación decrementa}

Donde $U R_{i}$ y $D R_{i}$ representan el límite de rampa ascendente y descendente del $i$-ésimo generador $(M W / h)$ y $P_{i}^{0}$ denota la potencia de salida anterior del $i$-ésimo generador. La combinación de (5) y (6) otorga el límite de potencia refinada para el $i$-ésimo generador como

$$
\max \left(P_{i}, \min , P_{i}^{0}-D R_{i}\right) \leq P_{i} \leq \min \left(P_{i, \max }, P_{i}^{0}-U R_{i}\right)
$$

\subsubsection{Zonas de operación prohibidas (ZOP)}

En [37], se aborda algunas razones que causan ZOP en la curva de costos de una unidad térmica, algunas de estas son, la introducción del temblor del cojinete del eje debido a una válvula de admisión de vapor, falla debido a la máquina o su equipo complementario asociado, como bomba, caldera, etc. Evitando el funcionamiento en ZOP se garantiza un mejor despacho. La potencia de salida entregada por el $i$-ésimo generador considerando ZOP se aprecia a continuación.

$$
P_{i} \in\left\{\begin{array}{c}
P_{i}^{\min } \leq P_{i} \leq P_{i, 1}^{L} \\
P_{i, k-1}^{U} \leq P_{i} \leq P_{i, k}^{L}\left(k=2,3, \ldots \ldots, N_{z i}\right) \\
P_{i, N_{i i}}^{U} \leq P_{i} \leq P_{i}^{\max }\left(k=N_{z i}\right)
\end{array}\right\}
$$


Donde $N_{z i}$ designa el número de ZOP en la curva de costo del $i$-ésimo generador, $k$ es el índice de ZOP del $i$-ésimo generador, $P_{i, k}^{L}$ y $P_{i, k}^{U}$ representan el límite inferior y superior de la $k$-ésima zona prohibida del $i$-ésimo generador.

\subsubsection{Restricciones de flujo de línea}

Para incorporar el efecto no deseado en las líneas de transmisión debido al flujo de potencia, se introduce la restricción de flujo de línea que se muestra a continuación.

$$
\left|P_{L f, k}\right| \leq P_{L f, k}^{\max } \quad k=l, \ldots \ldots \ldots, L
$$

Donde $P_{L f, k}$ y $P_{L f, k}^{\max }$ indican el flujo de potencia activa y el límite superior del flujo de potencia de la línea $k$ respectivamente, y $L$ representa el número de líneas de transmisión.

\subsubsection{Limitaciones de la reserva de giro del sistema}

La confiabilidad del SEP se mantiene mediante reservas adecuadas de giro. Es decir:

$$
\sum_{i=1}^{n} P_{i, \max } \geq P_{\text {Load }}+P_{\text {Loss }}+R
$$

El problema de DE en su forma más sencilla considera solo la restricción de balance de potencia activa (igualdad) y los límites de capacidad de generación (desigualdad). No obstante, cuantas más restricciones se utilicen más realista será el problema de DE.

\subsection{Problema de DE con múltiples máquinas de combustible (MMC)}

Las unidades generadoras pueden utilizar múltiples combustibles para su funcionamiento. Cada una de las unidades puede caracterizarse por varias funciones cuadráticas por partes. La función cuadrática por partes del -ésimo generador considerando MMC (despreciando los efectos CPV) se expresa por.

$$
\begin{gathered}
F_{i}\left(P_{i}\right)=\left\{\begin{array}{cc}
F_{i 1}\left(P_{i}\right), & \text { fuel } 1, P_{i \min } \leq P_{i} \leq P_{i 1} \\
F_{i 2}\left(P_{i}\right), & \text { fuel } 2, P_{i 1} \leq P_{i} \leq P_{i 2} \\
\vdots & \\
F_{i k}\left(P_{i}\right) & \text { fuel } k, P_{i k-1} \leq P_{i} \leq P_{\text {max }}
\end{array}\right. \\
\text { Donde } \begin{array}{c}
a_{i k}+b_{i k} P_{i}+c_{i k} P_{i}^{2} \\
F_{i}\left(P_{i}\right)=\begin{array}{c}
\text { if } P_{i \text { min. } k} \leq P_{i} \leq P_{i \max , k} \\
\text { fuel option } k, k=1,2, \ldots, k
\end{array}
\end{array}
\end{gathered}
$$

Donde $P_{i \min , k}$ y $P_{i \max , k}$ representan los límites mínimos y máximos de generación de potencia del generador $i$ con la opción de combustible $k$ respectivamente. $a_{i k}, b_{i k}$ y $c_{i k}$ se refieren a los coeficientes del costo de combustible del

generador $i$ para la opción de combustible $k$. El objetivo del problema de DE con MMC es reducir el costo total de combustible del generador sujeto a las limitaciones.

\subsection{Problema de DE con efecto CPV y MMC}

Para la resolución del problema de DE tomando en consideración el efecto CPV y MMC se debe incorporar estas simultáneamente en la función de costo de combustible. A continuación, se muestra la ecuación.

$$
F_{i}\left(P_{i}\right)=\begin{gathered}
a_{i k}+b_{i k} P_{i}+c_{i k} P_{i}^{2}+\left|e_{i k} * \operatorname{sen}\left(f_{i k} *\left(p_{i, \min }-p_{i}\right)\right)\right| \\
\text { if } P_{i \min , k} \leq P_{i} \leq P_{\text {max }, k} \\
\text { fuel option } k, k=1,2, \ldots, k
\end{gathered}
$$

\section{DESPACHO ECONÓMICO AMBIENTAL}

El [38] se menciona que la generación de energía en una central termoeléctrica se produce a partir de la quema de combustibles, renovables o no renovables. Entre los combustibles están el carbón mineral, gas natural, petróleo y biomasa. Como resultado de la generación eléctrica, se emiten impurezas del propio combustible, material particulado, dióxido de azufre $\left(\mathrm{SO}_{2}\right)$, óxido de nitrógeno $\left(\mathrm{NO}_{\mathrm{x}}\right)$ y dióxido de carbono $\left(\mathrm{CO}_{2}\right)$, que dañan la salud de los seres humanos, la fauna y la flora. Por lo tanto, es imprescindible que se controlen las emisiones contaminantes durante el proceso de generación de energía en las plantas termoeléctricas. Para reducir la contaminación del medio ambiente por estas emisiones, se utiliza una estrategia de despacho que minimiza la emisión de contaminantes atmosféricos, respetando las restricciones operativas del sistema y asegurando que se satisfaga la demanda. Esta estrategia se denomina problema de despacho económico ambiental (DEA).

En [17], se denota que los contaminantes ambientales como los óxidos de azufre $\mathrm{SO}_{x}$ y los óxidos de nitrógeno $\mathrm{NO}_{\mathrm{x}}$ causados por las unidades térmicas de combustibles fósiles pueden modelarse por separado. No obstante, a efectos de comparación, la emisión total de ton $/ h E\left(P_{G}\right)$ de estos contaminantes puede expresarse como.

$E\left(P_{G}\right)=\sum_{i=1}^{N} 10^{-2}\left(\alpha_{i}+\beta_{i} P_{G i}+\gamma_{i} P_{G i}^{2}\right)+\zeta_{1} \exp \left(\lambda_{i} P_{G i}\right)$

Donde $\alpha_{i}, \beta_{i}, \gamma_{i}, \zeta_{1}$ y $\lambda_{i}$ son coeficientes de las características de emisión del $i$-ésimo generador.

\section{METODOLOGÍA}

Los problemas ambientales que se manifiestan de las emisiones contaminantes producidas por las centrales eléctricas de combustibles fósiles se han convertido recientemente en un asunto inquietante, por ello, se ha considerado al DEA como la solución más viable para satisfacer la demanda del SEP. Para ayudar a los investigadores a desarrollar técnicas para abordar todos los problemas pendientes, este artículo presenta un estudio de las soluciones propuestas al problema de DEA.

Existen dos principales bases de datos que permiten acceder a la destacada producción científica y también difundirla, estás son: Web of Science (WOS) y SCOPUS. Hasta 
no hace mucho WOS era la única herramienta, internacional y multidisciplinaria, disponible para el acercamiento a la literatura de ciencia, tecnología, biomedicina y otras disciplinas. No obstante, desde hace unos años, a WOS le ha salido un contrincante de consideración SCOPUS, base de datos fundada por Elsevier S.L en 2004 que posee ciertas características que favorecen sobremanera al investigador. Para las revistas indexadas en ambas bases de datos, las de WOS presentar mayor factor de impacto; en cambio, aquellas que cubren solamente SCOPUS exteriorizan un factor de impacto menor que si están en ambas bases de datos [39]. Por ello, en el presente documento se ha realizado el análisis bibliométrico centrado principalmente en la base de datos de WOS.

La investigación se realizó en mayo del 2021 utilizando el término de búsqueda "Environmental Economic Dispatch". El refinamiento se realizó verificando el año de publicación, se ha tomado en consideración el lapso 20002020, ya que en WOS se registran artículos relacionados con la temática a partir de año 2000 , se han encontrado un total de 736 documentos y todos estos en lengua inglesa. En la Fig.1, se aprecia que la temática recibió una atención cada vez mayor y que se realizaron mayores investigaciones con el pasar de los años. El máximo número de artículos que se han publicado es de 126 en el año 2020, se estima que este número irá incrementando con el pasar del tiempo debido a la creciente conciencia pública sobre la protección del medio ambiente y la aprobación de las enmiendas de 1990 a la Ley de Aire Limpio de Estados Unidos [40] que obliga a las empresas de servicios públicos a modificar su diseño o estrategias operativas para minimizar la contaminación y las emisiones atmosféricas de las centrales eléctricas térmicas.

Fig. 1. TENDENCIA ANUAL DE LAS PUBLICACIONES EN WOS. (Publicaciones por año en WOS de la temática "Environmental Economic Dispatch")

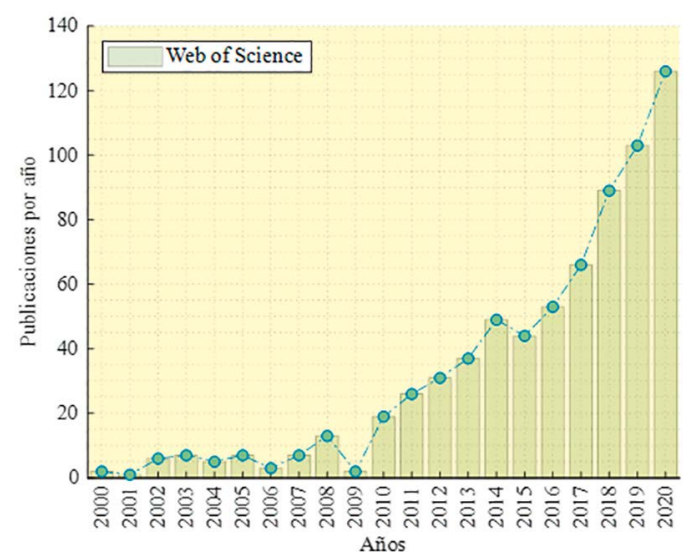

Fuente: Autores

Para saber los países que más indagan sobre la temática, en Vosviewer se ha elegido el tipo de análisis de citación y unidad de análisis de países, de esta manera los países que más han estado investigando son: China con 247 documentos y 4631 citaciones, Estados Unidos (USA) con 93 documentos y 3128 citaciones e Irán con 87 documentos y 2489 citaciones. Esta información fue extraída de la base de datos WOS y reflejada mediante el software VOSviewer. En Fig.2, se puede apreciar los 10 principales países que investigan sobre la temática de DEA.

Fig. 2. PAÍSES QUE INVESTIGAN EL PROBLEMA DE DEA SEGÚN WOS. (Los 10 principales países que investigan acerca del problema de DEA)

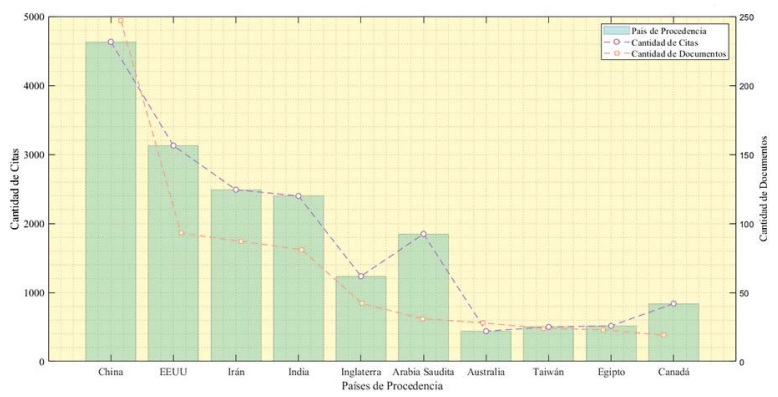

Fuente: Autores

Para conocer los autores que más indagan sobre la temática, en Vosviewer se ha elegido el tipo de análisis de citación y unidad de análisis de autores, así los autores que más han investigado en este campo son: Niknam, T con 10 documentos y 713 citaciones, Panigrahi, B con 9 documentos y 404 citaciones y Zhou, J con 9 documentos y 375 citaciones. Esta información fue extraída de la base de datos WOS y reflejada mediante el software VOSviewer. En Fig.3, se puede apreciar los 10 principales autores que investigan sobre la temática de DEA.

Fig. 3. AUTORES QUE INVESTIGAN EL PROBLEMA DE DEA SEGÚN WOS. (LoS 10 principales autores que investigan acerca del problema de DEA)

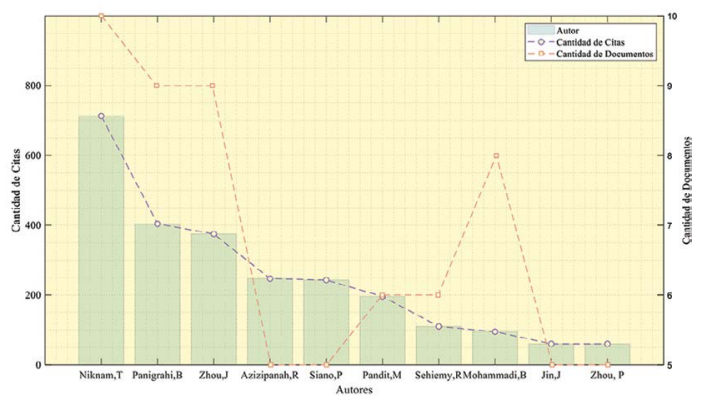

Fuente: Autores

Para saber las fuentes que más indagan sobre la temática, en Vosviewer se ha elegido el tipo de análisis de citación y unidad de análisis de fuentes, así las fuentes que más investigaciones poseen relacionadas a la temática son: Enegy con 56 documentos y 2220 citaciones, International Journal of Electrical Power \& Energy Systems (IJEPS) con 54 documentos y 2399 citaciones y Applied Energy (A. Energy) con 36 documentos y 962 citaciones. Esta información fue extraída de la base de datos WOS y reflejada mediante el software VOSviewer. En Fig.4, se puede apreciar las 10 principales fuentes que investigan sobre la temática de DEA, siendo las tres primeras las mencionadas anteriormente, $y$ en los puestos 
siguientes están las fuentes, Energies, Energy Conversion and Management (ECM), Electric Power Systems Research (EPSR), IEEE Access (IEEE A), Applied Soft Computing (ASC), let Generation Transmission \& Distribution (IETGTD) y Journal of Cleaner Production (JCP).

Fig. 4. FUENTES QUE INVESTIGAN EL PROBLEMA DE DEA SEGÚN WOS. (Las 10 principales fuentes que investigan acerca del problema de DEA)

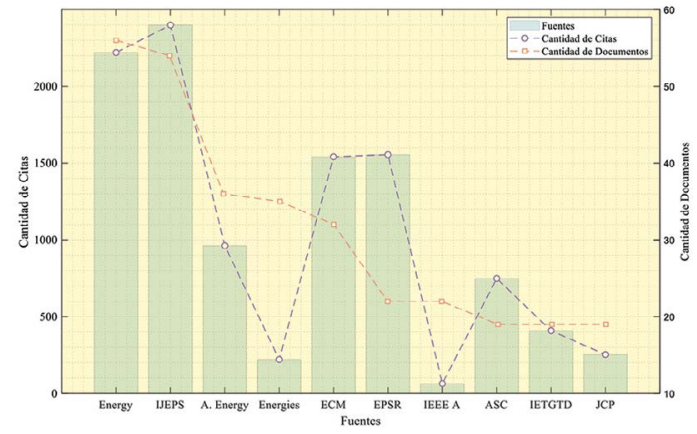

Fuente: Autores

Para conocer las organizaciones que más indagan sobre la temática, en Vosviewer se ha elegido el tipo de análisis de citación y unidad de análisis de organizaciones, de esta manera las organizaciones que más investigaciones poseen relacionadas a la temática son: North China Electric Power University (NCEPU) con 30 documentos y 298 citaciones, Islamic Azad University (IAU) con 29 documentos y 542 citaciones y Huazhong University Science \& Technology (HUS\&T) con 20 documentos y 712 citaciones. Esta información fue extraída de la base de datos WOS y reflejada mediante el software VOSviewer. En Fig.5, se puede apreciar las 10 principales organizaciones que investigan sobre la temática de DEA, siendo las tres primeras las mencionadas anteriormente, y en los puestos siguientes están las organizaciones, Tabriz University (Tabriz U), Tsinghua University (Tsinghua U), Shiraz University Technology (SUT), Iran University Science \& Technology (IUS\&T), Indian Institute Technology (IIT), Jadavpur University (JU) y Annamalai University (AU).

Fig. 5. ORGANIZACIONES QUE INVESTIGAN EL PROBLEMA DE DEA SEGÚN WOS. (Las 10 principales organizaciones que investigan acerca del problema de DEA)

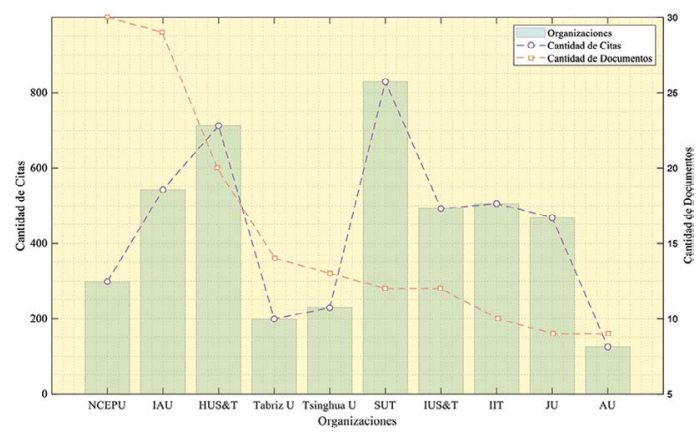

Fuente: Autores
Para conocer los documentos más relevantes dentro de la temática, en Vosviewer se ha elegido el tipo de análisis de citación y unidad de análisis de documentos, de esta manera los 15 documentos más citados en este campo se muestran en la Tabla I. Se aprecia que el documentos más citado es [41] con 926 citas. Se realizará el análisis de cada uno de estos y su aporte a la comunidad científica en la sección siguiente.

Tabla I.

15 DOCUMENTOS MÁS CITADOS SEGÚN WOS

(15 Documentos más citados)

\begin{tabular}{|l|l|l|}
\hline $\mathrm{N}^{\circ}$ & \multicolumn{1}{|c|}{ Documentos } & Citas \\
\hline 1 & $\begin{array}{l}\text { Environmental/ economic power dispatch using multi- } \\
\text { objective evolutionary algorithms }\end{array}$ & 926 \\
\hline 2 & $\begin{array}{l}\text { A novel multiobjective evolutionary algorithm or envi- } \\
\text { ronmental/ economic power dispatch }\end{array}$ & 479 \\
\hline 3 & $\begin{array}{l}\text { A niched pareto genetic algorithm for multiobjective en- } \\
\text { vironmental/ economic dispatch }\end{array}$ & 413 \\
\hline 4 & $\begin{array}{l}\text { Multiobjective evolutionary algorithms for electric power } \\
\text { dispatch problem }\end{array}$ & 778 \\
\hline 5 & $\begin{array}{l}\text { Multiobjective particle swarm optimization for environ- } \\
\text { mental/ economic dispatch problem }\end{array}$ & 424 \\
\hline 6 & $\begin{array}{l}\text { Multiobjective particle swarm algorithm with fuzzy clus- } \\
\text { tering for electrical power dispatch }\end{array}$ & 358 \\
\hline 7 & $\begin{array}{l}\text { Optimal allocation of ess in distribution systems with a } \\
\text { high penetration of wind energy }\end{array}$ & 504 \\
\hline 8 & $\begin{array}{l}\text { Economic environmental dispatch using multi-objective } \\
\text { differential evolution }\end{array}$ & 383 \\
\hline 9 & $\begin{array}{l}\text { Modeling, planning and optimal energy management } \\
\text { of combined cooling, heating, and power microgrid: a } \\
\text { review }\end{array}$ & 490 \\
\hline 10 & $\begin{array}{l}\text { Hourly coordination of electric vehicle operation and } \\
\text { volatile wind power generation in SCUC }\end{array}$ & 386 \\
\hline 11 & $\begin{array}{l}\text { Multi- objective operation management of a renewable } \\
\text { mg (micro-grid) with back-up micro-turbine/ fuel cell/ } \\
\text { battery hybrid power source }\end{array}$ & 466 \\
\hline 12 & $\begin{array}{l}\text { Applications of fuzzy logic in renewable energy sys- } \\
\text { tems - a review }\end{array}$ & 383 \\
\hline 13 & $\begin{array}{l}\text { Impacts of wind power on thermal generation unit com- } \\
\text { mitment and dispatch }\end{array}$ & 797 \\
\hline 14 & $\begin{array}{l}\text { Deep learning-based ensemble approach for probabi- } \\
\text { listic wind power forecasting }\end{array}$ & 408 \\
\hline 15 & $\begin{array}{l}\text { Multi-objective scheduling of electric vehicles in smart } \\
\text { distribution system }\end{array}$ & 275 \\
\hline
\end{tabular}

\section{REVISIÓN ARTÍCULOS RELEVANTES}

Abido, M.A. en [41] propone un nuevo enfoque basado en el algoritmo evolutivo de Pareto de fuerza (SPEA) para manejar el DEA como un verdadero problema de optimización multiobjetivo con objetivos competitivos y no medibles. El enfoque propuesto emplea un mecanismo de preservación de la diversidad para superar los problemas de sesgo de búsqueda y convergencia prematura. También se impone un algoritmo de agrupamiento jerárquico para proporcionar al tomador de decisiones un conjunto de Pareto-óptimo representativo y manejable. Además, se emplea la teoría de conjuntos difusos para extraer la mejor solución no dominada de compromiso. Se han llevado a cabo varias ejecuciones de optimización del enfoque propuesto en un sistema de 
prueba estándar. Los resultados demuestran las capacidades del enfoque propuesto para generar soluciones óptimas de Pareto bien distribuidas del problema de DEA multiobjetivo en una sola ejecución. La comparación con las técnicas clásicas demuestra la superioridad del enfoque propuesto y confirma su potencial para resolver el problema de DEA multiobjetivo. Además, la extensión del enfoque propuesto para incluir más objetivos es un proceso sencillo.

Abido, M.A. en [42] a novel multiobjective evolutionary algorithm for environmental/economic power dispatch (EED presenta un nuevo algoritmo evolutivo multiobjetivo para el problema de optimización de DEA. Se propone un nuevo enfoque basado en un algoritmo genético de clasificación no dominado para manejar el problema como un verdadero problema de optimización multiobjetivo con objetivos competitivos y no conmensurables. El enfoque propuesto emplea un mecanismo de preservación de la diversidad para superar la convergencia prematura y los problemas de sesgo de búsqueda y producir un conjunto óptimo de Pareto bien distribuido de soluciones no dominadas. También se impone un algoritmo de agrupamiento jerárquico para proporcionar al tomador de decisiones un conjunto óptimo de Pareto representativo y manejable. Además, se emplea la teoría de conjuntos difusos para extraer la mejor solución de compromiso sobre la curva de compensación. Se llevan a cabo varias ejecuciones de optimización del enfoque propuesto en el sistema de prueba estándar IEEE 30-bus. Los resultados demuestran las capacidades del enfoque propuesto para generar soluciones no dominadas óptimas de Pareto verdaderas y bien distribuidas del problema de DEA multiobjetivo en una sola ejecución. Los resultados de la simulación con el enfoque propuesto se han comparado con los reportados en la literatura. La comparación demuestra la superioridad del enfoque propuesto y confirma su potencial para resolver el problema de DEA multiobjetivo.

Abido, M.A. en [18] presenta un enfoque basado en el algoritmo genético de Pareto de níquel (NPGA) para resolver el problema de DEA de múltiples objetivos. El enfoque propuesto basado en NPGA maneja el problema como un problema de objetivos múltiples con objetivos de emisiones y costos competitivos y no conmensurables. Una de las principales ventajas del enfoque propuesto es que no hay restricciones en el número de objetivos optimizados. El enfoque propuesto tiene un mecanismo de preservación de la diversidad para superar el problema de la convergencia prematura. Se desarrolla e impone un algoritmo de agrupamiento jerárquico para proporcionar al tomador de decisiones un conjunto óptimo de Pareto representativo y manejable. Además, se emplea la teoría de conjuntos difusos para extraer la mejor solución de compromiso. Se llevan a cabo varias ejecuciones de optimización del enfoque propuesto en el sistema de prueba estándar IEEE 30-bus. Los resultados demuestran las capacidades del enfoque propuesto para generar soluciones no dominadas óptimas de Pareto bien distribuidas del problema de EED multiobjetivo en una sola ejecución. La comparación con los métodos clásicos demuestra la su- perioridad del enfoque propuesto y confirma su potencial para resolver el problema de EED multiobjetivo.

Abido, M.A. en [43] evalúa exhaustivamente la potencialidad y la efectividad de los algoritmo evolutivos multiobjetivos basados en Pareto (MOEA). Específicamente, el algoritmo genético de clasificación no dominado, el algoritmo genético de Pareto con níquel y el algoritmo evolutivo de Pareto de fuerza (SPEA) se han desarrollado y aplicado con éxito a un problema de despacho de energía eléctrica ambiental / económico. En este trabajo se propone un nuevo procedimiento para medir la calidad con el fin de evaluar diferentes técnicas. Se ha desarrollado un procedimiento de verificación de viabilidad y se ha superpuesto al MOEA para restringir la búsqueda a la región factible del espacio problemático. También se impone un algoritmo de agrupamiento jerárquico para proporcionar al operador del sistema de energía un conjunto óptimo de Pareto representativo y manejable. Además, se desarrolla un enfoque basado en la teoría de conjuntos difusos para extraer una de las soluciones óptimas de Pareto como la mejor solución de compromiso. Estos algoritmos evolutivos multiobjetivos se han examinado individualmente y se han aplicado al sistema de prueba estándar de seis generadores de bus 30 de IEEE. Se han llevado a cabo varias corridas de optimización en diferentes casos de complejidad del problema. Los resultados de MOEA se han comparado con los reportados en la literatura. Los resultados confirman el potencial y la eficacia de MOEA en comparación con las técnicas tradicionales de optimización multiobjetivo. Además, los resultados demuestran la superioridad del SPEA como un algoritmo evolutivo multiobjetivo prometedor para resolver la optimización multiobjetivo de diferentes sistemas de energía.

Abido, M.A. en [17] propone una nueva técnica de optimización de enjambres de partículas multiobjetivo (MOPSO) para el problema de DEA. La técnica MOPSO propuesta desarrolla una versión multiobjetivo de PSO al proponer la redefinición de los mejores individuos globales y locales en el dominio de la optimización multiobjetivo. La técnica MOPSO propuesta se ha implementado para resolver el problema de DEA con objetivos de emisiones y costes competitivos y no conmensurables. Se han llevado a cabo varias ejecuciones de optimización del enfoque propuesto en un sistema de prueba estándar. Los resultados demuestran las capacidades de la técnica MOPSO propuesta para generar un conjunto de soluciones óptimas de Pareto bien distribuidas en una sola ejecución. La comparación con las diferentes técnicas reportadas demuestra la superioridad del MOPSO propuesto en términos de la diversidad de las soluciones óptimas de Pareto obtenidas. Además, se implementa una medida de calidad de las soluciones óptimas de Pareto donde los resultados confirman el potencial de la técnica MOPSO propuesta para resolver el problema de DEA multiobjetivo y producir soluciones no dominadas de alta calidad.

Agrawal, S. y otros en [44] proponen un algoritmo de enjambre de partículas basado en agrupamiento difuso (FCPSO) para resolver el problema de DEA altamente limitado que 
implica propósios en conflicto. FCPSO emplea un repositorio externo para preservar las partículas no dominadas que se encuentran a lo largo del proceso de búsqueda. La técnica de agrupamiento difuso propuesta gestiona el tamaño del repositorio dentro de los límites sin destruir las características del frente de Pareto. Se ha ingresado un mecanismo de Niching para dirigir las partículas hacia regiones menos exploradas del frente de Pareto. Para evitar el atrapamiento en óptimas locales y mejorar la capacidad exploratoria de las partículas, se ha propuesto un operador de mutación autoadaptativo. Además, el algoritmo incorpora un mecanismo de retroalimentación basado en datos difusos y utiliza iterativamente la información para determinar la solución de compromiso. El rendimiento del algoritmo ha sido examinado sobre el sistema de prueba de seis generadores de bus estándar IEEE 30, mediante el cual generó un frente de Pareto distribuido uniformemente cuya optimización ha sido autenticada mediante evaluación comparativa contra el método de restricción.

Atwa, Y. y El- Saadany, E. en [45] exponen que las preocupaciones ambientales y las incertidumbres en el costo del combustible asociadas con el uso de fuentes de energía convencionales han dado como resultado un rápido crecimiento en la cantidad de energía eólica conectada a las redes de distribución. Y propone una metodología para la asignación de un sistema de almacenamiento de energía (ESS) en un sistema de distribución con alta penetración de energía eólica. El objetivo final es maximizar los beneficios tanto para el propietario de la generación distribuida como para la empresa de servicios públicos dimensionando el ESS para acomodar todas las cantidades de energía eólica derramada y luego asignándola dentro del sistema para minimizar el costo anual de la electricidad.

Basu, M. [46] propone la evolución diferencial multiobjetivo para resolver el problema de DEA. Los resultados numéricos de tres sistemas de prueba demuestran las capacidades del enfoque propuesto. El problema se ha formulado como una optimización multiobjetivo con objetivos competitivos de costes de combustible y emisiones. Los resultados obtenidos con el enfoque propuesto se han comparado con los obtenidos a partir de la evolución diferencial de Pareto. De la comparación se ve que el enfoque propuesto proporciona un rendimiento competitivo en términos de solución y tiempo de cálculo. La evolución diferencial multiobjetivo propuesta es simple, robusta y eficiente. No impone ninguna limitación al número de objetivos y puede ampliarse para incluir más objetivos.

Gu, W. y otros en [47] presentan una revisión general del modelado, la planificación y la gestión energética de la microrred combinada de refrigeración, calefacción y energía (CCHP). El rendimiento de una microrred CCHP desde los puntos de vista técnico, económico y medioambiental dependen en gran medida del diseño de la microrred y la gestión energética. El modelado preciso es el primer y más importante paso para la planificación y la gestión energética de la microrred de CCHP, por lo que este documento presenta primero una revisión del modelado de la microrred de CCHP. Con respecto a la planificación de la microrred CCHP, se dan varios métodos de evaluación e indicadores ampliamente aceptados para los sistemas de cogeneración. Luego se presentan los esfuerzos de investigación sobre los métodos de planificación de la microrred de CCHP. Finalmente, se revisa brevemente la gestión energética de la microrred CCHP en términos de desacoplamiento de cogeneración, estrategias de control, reducción de emisiones y métodos de resolución de problemas.

Khodayar, M. y otros en [48] the coordinated integration of aggregated plug-in electric vehicle (PEV estudian la integración coordinada de flotas agregadas de vehículos eléctricos enchufables (PEV) y fuentes de energía renovable (energía eólica) en los sistemas de energía mediante el modelo estocástico de compromiso de unidad con restricciones de seguridad (Stochastic SCUC), que minimiza el esperado costo de operación de la red considerando el comportamiento aleatorio de los muchos PEV. Los PEV son dispositivos móviles y distribuidos con opciones diferibles para el suministro / uso de energía en distintos momentos y ubicaciones. La mayor utilización de los PEV, que consumen electricidad en lugar de combustibles fósiles para conducir, ofrece oportunidades económicas y ambientales únicas y plantea nuevos desafíos para la operación y planificación del sistema de energía eléctrica. La capacidad de almacenamiento de los PEV podría ayudar a los sistemas de energía a mitigar la variabilidad de las fuentes de energía renovables y reducir los costos de operación de la red. Vehicle-to-grid (V2G) permite que los PEV tengan flujos de energía bidireccionales una vez que están conectados a la red, es decir, pueden inyectar energía y extraer energía de la red, lo que agrega mayor complejidad a las operaciones del sistema eléctrico. Los PEV significan el comportamiento aleatorio de los clientes al considerar sus patrones de conducción, los requisitos energéticos de ubicación, las interconexiones de redes topológicas y otras limitaciones impuestas por los consumidores. Las pruebas numéricas demuestran la efectividad del enfoque propuesto para analizar el impacto de los PEV en el costo de operación de la red y el despacho de energía eólica por hora.

Moghaddam, A. y otros en [49] proponen un algoritmo de optimización adaptativa de enjambre de partículas modificadas (AMPSO) multiobjetivo experto para el funcionamiento óptimo de una microred (MG) típico con fuentes de energía renovable (RES) acompañado de una fuente de energía híbrida de respaldo de micro turbina / pila de combustible / batería. para nivelar el desajuste de energía o para almacenar el excedente de energía cuando sea necesario. El problema se formula como un problema de optimización multiobjetivo de restricción no lineal para minimizar el costo operativo total y la emisión neta simultáneamente. Para mejorar el proceso de optimización, se utiliza un algoritmo PSO híbrido basado en un mecanismo Chaotic Local Search (CLS) y una estructura Fuzzy Self Adaptive (FSA). El algoritmo propuesto se prueba en un MG típico y su rendimiento superior se compara con los de otros algoritmos evolutivos como algoritmo genético (GA) y optimización de enjambre de partículas (PSO). 
Suganthi, L. y otros en [50] exponen que el modelado y la planificación energética son vitales para la prosperidad económica futura y la seguridad ambiental. En este contexto, existen técnicas de computación como la lógica difusa, las redes neuronales y los algoritmos genéticos modelados de energía para mapear con precisión los sistemas de energía. En este documento, se ha revisado las aplicaciones de modelos basados en lógica difusa en sistemas de energía renovable, a saber, aplicaciones solares, eólicas, bioenergéticas, microrredes e híbridas. Se encuentra que los modelos basados en lógica difusa se utilizan ampliamente en los últimos años para la evaluación del sitio, para la instalación de parques fotovoltaicos / eólicos, seguimiento de puntos de energía en energía solar fotovoltaica / eólica, optimización entre criterios conflictivos. La indagación indica que los modelos basados en lógica difusa proporcionan estimaciones realistas.

Ummels, B. y otros en [51] proponen un nuevo método de simulación que puede evaluar completamente los impactos de la energía eólica a gran escala en las operaciones del sistema desde el costo, la confiabilidad y las perspectivas ambientales. El método utiliza una serie de tiempo de velocidades del viento promedio de 15 minutos observadas y pronosticadas en ubicaciones previstas de parques eólicos terrestres y marinos. La herramienta UnitCommitment and Economic Dispatch (UC-ED) está adaptada para permitir revisiones frecuentes de los horarios de las unidades de generación convencionales, utilizando información sobre la producción actual de energía eólica y las previsiones para las próximas $36 \mathrm{~h}$. Esta se considera la forma más fiel de simular operaciones reales y actividades de planificación a corto plazo para un sistema con una gran penetración de energía eólica. La formulación del problema incluye restricciones de velocidad de rampa para los programas de generación y para la activación de la reserva, y el tiempo mínimo de actividad y tiempo de inactividad de las unidades convencionales. Se muestran los resultados para un escenario futuro realista del sistema eléctrico holandés. Se muestra que problemas como la regulación y reserva de energía insuficientes, que normalmente se asocian con la variabilidad y la previsibilidad limitada de la energía eólica, solo pueden evaluarse junto con las características específicas del sistema de generación convencional en el que se integra la energía eólica. Para el sistema térmico con una gran proporción de calor y energía combinados (CHP) investigado aquí, la previsión de energía eólica no proporciona beneficios significativos para un compromiso y despacho óptimos de la unidad. Se producen problemas de carga mínima, que dan como resultado una pérdida de viento en cantidades que aumentan con la energía eólica instalada.

Wang, H. y otros en [52] proponen un enfoque de conjunto basado en el aprendizaje para la predicción probabilística de energía eólica. En este enfoque, se plantea originalmente un método avanzado de pronóstico de puntos basado en la transformada de wavelet y convolucional red neuronal. La transformada wavelet se utiliza para descomponer los datos brutos de energía eólica en diferentes frecuencias. Las características no lineales en cada frecuencia que se utilizan para mejorar la precisión del pronóstico son más tarde aprendidas de manera efectiva por la red neuronal convolucional. Las incertidumbres en los datos de energía eólica, es decir, la especificación incorrecta del modelo y el ruido de datos se identifican por separado a partir de entonces. En consecuencia, la distribución probabilística de los datos de energía eólica se puede formular estadísticamente. El conjunto propuesto se ha evaluado ampliamente utilizando datos reales de parques eólicos de China, y los resultados demuestran que las incertidumbres en los datos de energía eólica se pueden aprender mejor utilizando el enfoque propuesto $y$ que se obtenga un desempeño competitivo.

Zakariazadeh, A. y otros en [53] exponen que al prepararse para la adopción generalizada de vehículos eléctricos $(\mathrm{EV})$, un tema importante es utilizar un modelo de programación de carga / descarga de vehículos eléctricos adecuado que sea capaz de considerar simultáneamente los objetivos económicos y ambientales, así como las limitaciones técnicas de las redes de distribución. Este documento propone un método de programación operacional multiobjetivo para la carga / descarga de vehículos eléctricos en un sistema de distribución inteligente. El marco multiobjetivo propuesto, basado en el método de restricción electrónica aumentada, tiene como objetivo minimizar los costos operativos y las emisiones totales. La capacidad Vehicle to Grid (V2G), así como los patrones reales de los conductores, se consideran para generar las soluciones óptimas de Pareto. La técnica de descomposición de Benders se utiliza para resolver el modelo de optimización propuesto y convertir el problema no lineal de enteros mixtos a gran escala en problemas de programación lineal de enteros mixtos y de programación no lineal. La eficacia del enfoque de programación de recursos propuesto se prueba en un sistema de prueba de distribución de 33 buses durante un período de 24 horas. Los resultados muestran que el método de carga / descarga de los vehículos eléctricos propuestos puede reducir tanto el costo de operación como las emisiones contaminantes del aire.

Estos 15 documentos analizados han sido elegidos debido a que representan un mayor grado de relevancia y alcance al problema identificado en esta investigación, en la Tabla II se aprecia a modo de resumen la temática que aborda el documento y la metodología propuesta, además de datos importantes del documento, tales como, el año de publicación, el tema, la revista de indexación y el número de citas.

Se aprecia que [41] figura como el documento más citado con 926 citas, su año de publicación fue en el 2003 y la revista de indexación es la IEEE Transactions on Power Systems. Además, esta revista es la que más documentos posee de los 15 analizados, tiene en su repertorio 5 documentos representando un $33.33 \%$ del total. Se puede notar que [48] representa el documento más completo de todos ya que en su contenido se aborda todas las temáticas, siendo estas, despacho económico ambiental, energía renovable y vehículos eléctricos. 


\begin{tabular}{|c|c|c|c|c|c|c|c|c|c|c|c|c|c|c|c|c|c|}
\hline \multirow{12}{*}{ 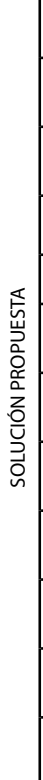 } & 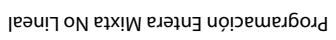 & & & & & & & & & & & & & & & $\times$ & - \\
\hline & 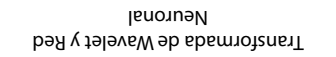 & & & & & & & & & & & & & & $\times$ & & - \\
\hline & 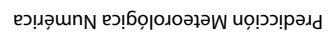 & & & & & & & & & & & & & $\times$ & & & - \\
\hline & 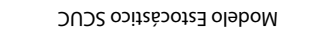 & & & & & & & & & & $x$ & & & & & & - \\
\hline & 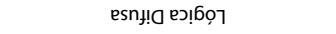 & & & & & & & & & $x$ & & & $\times$ & & & & n \\
\hline & 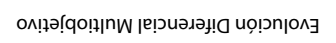 & & & & & & & & $\times$ & & & & & & & & - \\
\hline & 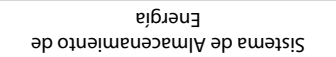 & & & & & & & $\times$ & & & & & & & & & - \\
\hline & 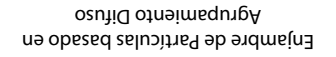 & & & & & & $\times$ & & & & & & & & & & - \\
\hline & 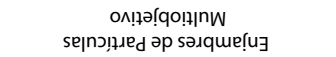 & & & & & $\times$ & & & & & & $\times$ & & & & & n \\
\hline & 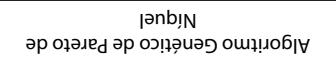 & & & $x$ & $x$ & & & & & & & & & & & & a \\
\hline & 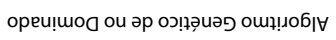 & & $x$ & & $x$ & & & & & & & & & & & & n \\
\hline & 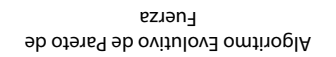 & $\times$ & & & $x$ & & & & & & & & & & & & n \\
\hline \multirow{4}{*}{ 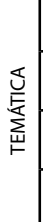 } & soว!!క!!, & $\times$ & $\times$ & $\times$ & $\times$ & $x$ & $\times$ & & $\times$ & $x$ & & $\times$ & & $\times$ & $\times$ & & $=$ \\
\hline & 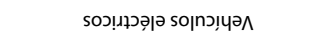 & & & & & & & & & & $x$ & & & & & $x$ & u \\
\hline & әүqелоиәу е̣бљәиэ & & & & & & & $x$ & & $x$ & $x$ & $x$ & $\times$ & $\times$ & $\times$ & & n \\
\hline & 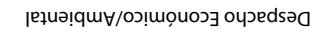 & $\times$ & $x$ & $\times$ & $\times$ & $\times$ & $\times$ & $\times$ & $\times$ & $x$ & $\times$ & $x$ & & & & $\times$ & $\simeq$ \\
\hline \multirow{5}{*}{ 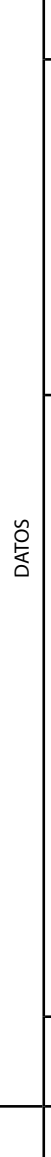 } & 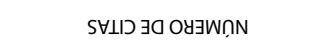 & హั & જે & $\stackrel{m}{\sigma}$ & $\stackrel{\infty}{\gtrless}$ & 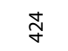 & $\stackrel{\infty}{m}$ & ¿̊̀ & $\stackrel{m}{m}$ & \& & $\stackrel{\infty}{m}$ & \& & $\stackrel{m}{\infty}$ & $\hat{\hat{n}}$ & $\stackrel{\infty}{\sigma}$ & $\stackrel{n}{\sim}$ & 䙲 \\
\hline & 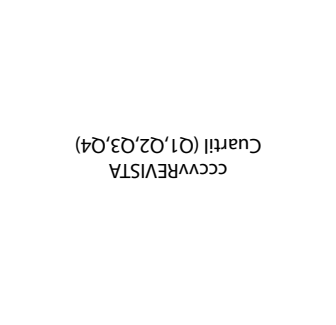 & 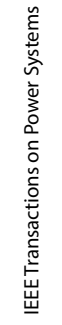 & 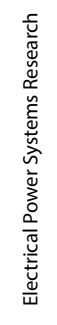 & 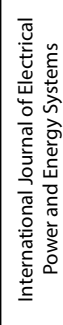 & 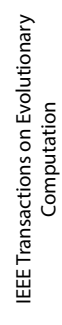 & 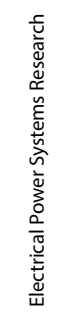 & 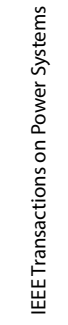 & 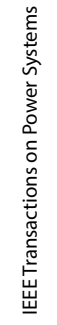 & 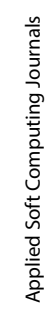 & 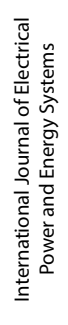 & 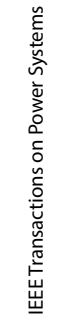 & $\begin{array}{l}\text { जे } \\
\text { एँ } \\
\text { w }\end{array}$ & 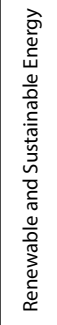 & 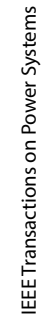 & 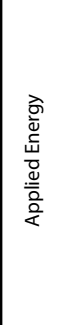 & 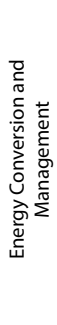 & \\
\hline & 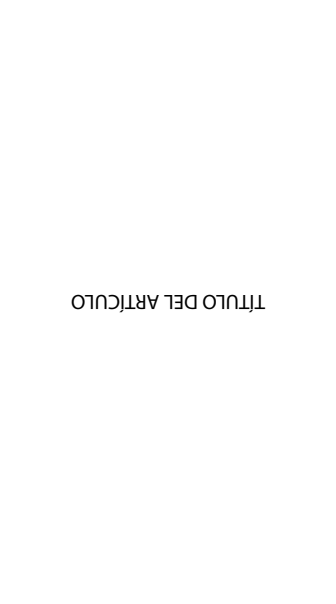 & 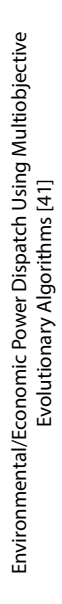 & 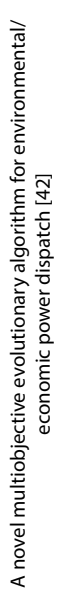 & 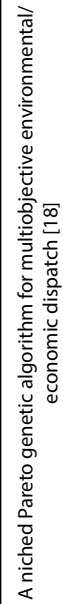 & 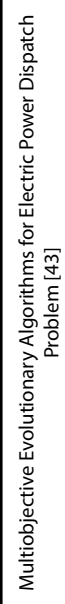 & 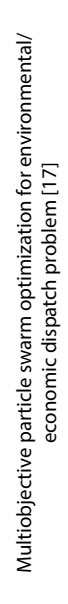 & 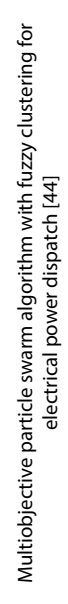 & 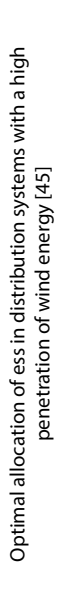 & 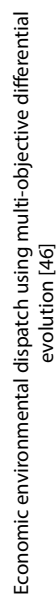 & 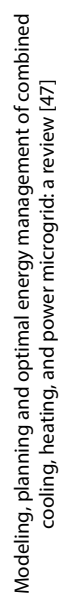 & 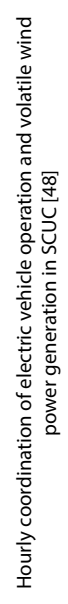 & 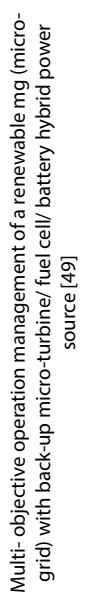 & 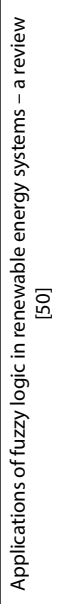 & 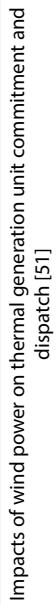 & 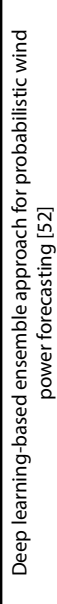 & 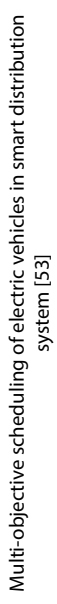 & \\
\hline & 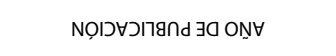 & 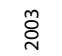 & $\stackrel{m}{\circ}$ & $\stackrel{m}{\circ}$ & ঃั̀ & 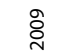 & 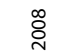 & $\frac{\circ}{2}$ & $\overline{\bar{n}}$ & 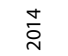 & $\stackrel{̃}{i}$ & $\bar{n}$ & $\stackrel{n}{\frac{n}{n}}$ & 离 & $\hat{\check{n}}$ & $\stackrel{+}{\circ}$ & \\
\hline & Wझ॥I & - & N & $m$ & 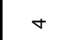 & in & 0 & n & $\infty$ & & 우 & $=$ & $\simeq$ & $\underline{m}$ & \pm & $\stackrel{n}{\sim}$ & \\
\hline
\end{tabular}


De todas las temáticas analizadas la producción de contenido de vehículos eléctricos y su relación con DEA es la más baja representando un $13.33 \%$. Mientras que las temáticas de despacho económico ambiental y métodos metaheurísticos son las más analizadas con un $80 \%$ y $73.33 \%$ respectivamente. Por otro lado, la temática de energía renovables se encuentra con un $46.66 \%$. Existen un total de 12 soluciones propuestas para solventar el problema de DEA, esto otorga una perspectiva amplia de resolución. Existe un máximo de 2 veces que se utiliza una misma propuesta de solución, esto indica que los documentos indagados son variados y poseen metodologías nuevas que ayudan sobremanera en el tema de investigación.

\section{CONCLUSIONES}

Existen dos principales bases de datos que permiten acceder a la destacada producción científica y también difundirla, estás son: Web of Science (WOS) y SCOPUS. En el presente documento se ha realizado el análisis bibliométrico tomando en consideración WOS, ya que, este presenta un factor de impacto mayor. La investigación se realizó en mayo del 2021 utilizando el término de búsqueda "Environmental Economic Dispatch". El refinamiento se realizó verificando el año de publicación, se ha tomado en consideración el lapso 2000-2020, ya que en WOS se registran artículos relacionados con la temática a partir de año 2000 , se han encontrado un total de 736 documentos y todos estos en lengua inglesa. Esta temática recibe una atención cada vez mayor y se realizaran mayores investigaciones con el pasar de los años esto se debe a la creciente conciencia pública sobre la protección del medio ambiente y la aprobación de las enmiendas de 1990 a la Ley de Aire Limpio de Estados Unidos que obliga a las empresas de servicios públicos a modificar su diseño o estrategias operativas para minimizar la contaminación y las emisiones atmosféricas de las centrales eléctricas térmicas.

Para realizar la revisión literaria, se ha realizado un análisis bibliométrico para obtener los 15 documentos más relevantes dentro de la temática de investigación. Estos documentos analizados han sido estadísticamente significativos con resultados positivos que pueden ayudar sobremanera a resolver el problema de despacho económico/ ambiental (DEA). En este contexto, se obtuvo que [48] the coordinated integration of aggregated plug-in electric vehicle (PEV representa el documento más completo de todos ya que en su contenido se aborda las temáticas de DEA, energía renovable y vehículos eléctricos. Por otro lado, [41] figura como el documento más citado con 926 citas y aborda las temáticas de DEA y métodos metaheurísticos. Este artículo será una guía útil para los investigadores.

\section{REFERENCIAS}

[1] G. Abbas, J. Gu, U. Farooq, M. U. Asad, and M. El-Hawary, "Solution of an Economic Dispatch Problem Through Particle Swarm Optimization: A Detailed Survey - Part I," IEEE Access, vol. 5, pp. 15105-15141, 2017, doi: 10.1109/ ACCESS.2017.2723862.
[2] G. Wood, Allen; Wollenberg, Bruce; Sheblé, Power Generation, Operation and Control, Third Edit. Ney Jersey: IEEE Canada, 2013.

[3] J. K. Arthur, E. A. Frimpong, and J. O. Adjei, "A Literature Survey of Recent Advances in the Solution of Combined Economic Emission Dispatch Problem," IEEE AFRICON Conf., vol. 2019-Septe, 2019, doi: 10.1109/AFRICON46755.2019.9133761.

[4] L. Wang and C. Singh, "Environmental/Economic Power Dispatch Using a Fuzzified Multi-Objective Particle Swarm Optimization Algorithm," Electr. Power Syst. Res., vol. 77, no. 12, pp. 1654-1664, Oct. 2007, doi: 10.1016/j.epsr.2006.11.012.

[5] S. P. Simon and S. Hemamalini, "Maclaurin series-based Lagrangian method for economic dispatch with valve-point effect," IET Gener. Transm. Distrib., vol. 3, no. 9, pp. 859-871, Sep. 2009, doi: 10.1049/iet-gtd.2008.0499.

[6] R. Chakrabarti, P. K. Chattopadhyay, and C. K. Panigrahi, "A review of recent advances in dynamic economic dispatch," J. Inst. Eng. Electr. Eng. Div., vol. 91, no. JUNE, pp. 9-15, 2010.

[7] W. M. Lin and S. J. Chen, "Bid-based dynamic economic dispatch with an efficient interior point algorithm," Int. J. Electr. Power Energy Syst., vol. 24, no. 1, pp. 51-57, 2002, doi: 10.1016/S0142-0615(01)00007-2.

[8] J.P.Zhan, Q. H. Wu, C. X. Guo, and X.X. Zhou, "Fast \$Vlambda\$Iteration Method for Economic Dispatch With Prohibited Operating Zones," IEEE Trans. Power Syst., vol. 29, no. 2, pp. 990-991, Mar. 2014, doi: 10.1109/TPWRS.2013.2287995.

[9] S.-D. Chen and J.-F. Chen, "A direct Newton-Raphson economic emission dispatch," Int. J. Electr. Power Energy Syst., vol. 25, no. 5, pp. 411-417, Jun. 2003, doi: 10.1016/S0142-0615(02)00075-3.

[10] A. Farag, S. Al-Baiyat, and T. C. Cheng, "Economic load dispatch multiobjective optimization procedures using linear programming techniques," IEEE Trans. Power Syst., vol. 10, no. 2, pp. 731-738, 1995, doi: 10.1109/59.387910.

[11] A. M. Sasson and G. J. Fisher, "Nonlinear Programming Solutions for Load-Flow, Minimum-Loss, and Economic Dispatching Problems," IEEE Trans. Power Appar. Syst., vol. PAS88, no. 4, pp. 399-409, 1969, doi: 10.1109/TPAS.1969.292460.

[12] L. G. Papageorgiou and E. S. Fraga, "A mixed integer quadratic programming formulation for the economic dispatch of generators with prohibited operating zones," Electr. Power Syst. Res., vol. 77, no. 10, pp. 1292-1296, 2007, doi: 10.1016/j.epsr.2006.09.020.

[13] W. R. Barcelo and P. Rastgoufard, "Dynamic economic dispatch using the extended security constrained economic dispatch algorithm," IEEE Trans. Power Syst., vol. 12, no. 2, pp. 961-967, 1997, doi: 10.1109/59.589791.

[14] F. N. Lee and A. M. Breipohl, "Reserve Constrained Economic Dispatch with Prohibited Operating Zones," IEEE Trans. Power Syst., vol. 8, no. 1, pp. 246-254, 1993, doi: 10.1109/59.221233.

[15] J. Y. Fan and J. D. McDonald, "A practical approach to real time economic dispatch considering unit's prohibited operating zones," IEEE Trans. Power Syst., vol. 9, no. 4, pp. 1737-1743, 1994, doi: 10.1109/59.331425.

[16] S. Hemamalini and S. P. Simon, "Dynamic economic dispatch using Maclaurin series based Lagrangian method," Energy Convers. Manag., vol. 51, no. 11, pp. 2212-2219, 2010, doi: 10.1016/j.enconman.2010.03.015.

[17] M. A. Abido, "Multiobjective Particle Swarm Optimization for Environmental/Economic Dispatch Problem," Electr. Power Syst. Res., vol. 79, no. 7, pp. 1105-1113, Jul. 2009, doi: 10.1016/j.epsr.2009.02.005. 
[18] M. A. Abido, "A Niched Pareto Genetic Algorithm for Multiobjective Environmental/Economic Dispatch," Int. J. Electr. Power Energy Syst., vol. 25, no. 2, pp. 97-105, 2003, doi: 10.1016/S0142-0615(02)00027-3.

[19] K. Xu, J. Zhou, Y. Zhang, and R. Gu, “Differential evolution based on $\varepsilon$-domination and orthogonal design method for power environmentally-friendly dispatch," Expert Syst. Appl., vol. 39, no. 4, pp. 3956-3963, Mar. 2012, doi: 10.1016/j.eswa.2011.08.145.

[20] T. Jayabarathi, K. Jayaprakash, D. N. Jeyakumar, and T. Raghunathan, "Evolutionary programming techniques for different kinds of economic dispatch problems," Electr. Power Syst. Res., vol. 73, no. 2, pp. 169-176, 2005, doi: 10.1016/j.epsr.2004.08.001.

[21] M. Vanitha, "Non Convex Economic Load Dispatch Problem By Efficient Biogeography Based Optimization Algorithm," Intell. Syst. Ref. Libr., vol. 62, pp. 81-91, 2014, doi: 10.1007/978-3-319-03404-1_5.

[22] K. Vishwakarma, H. Dubey, M. Pandit, and B. Panigrahi, "Simulated annealing approach for solving economic load dispatch problems with valve point loading effects," Int. J. Eng. Sci. Technol., vol. 4, no. 4, pp. 60-72, 2013, doi: 10.4314/ijest.v4i4.6.

[23] S. Pothiya, I. Ngamroo, and W. Kongprawechnon, "Ant colony optimisation for economic dispatch problem with nonsmooth cost functions," Int. J. Electr. Power Energy Syst., vol. 32, no. 5, pp. 478-487, 2010, doi: 10.1016/j.ijepes.2009.09.016.

[24] T. Niknam, H. D. Mojarrad, H. Z. Meymand, and B. B. Firouzi, "A new honey bee mating optimization algorithm for nonsmooth economic dispatch," Energy, vol. 36, no. 2, pp. 896908, 2011, doi: 10.1016/j.energy.2010.12.021.

[25] B. K. Panigrahi and V. Ravikumar Pandi, "Bacterial foraging optimisation: Nelder-Mead hybrid algorithm for economic load dispatch," IET Gener. Transm. Distrib., vol. 2, no. 4, p. 556, 2008, doi: 10.1049/iet-gtd:20070422.

[26] M. H. Sulaiman, M. W. Mustafa, Z. N. Zakaria, O. Aliman, and S. R. Abdul Rahim, "Firefly algorithm technique for solving economic dispatch problem," 2012 IEEE Int. Power Eng. Optim. Conf. PEOCO 2012 - Conf. Proc., no. June, pp. 90-95, 2012, doi: 10.1109/PEOCO.2012.6230841.

[27] N.T. P. Thao and N. T. Thang, "Environmental Economic Load Dispatch with Quadratic Fuel Cost Function Using Cuckoo Search Algorithm," Int. J. u- e-Service, Sci. Technol., vol. 7, no. 2, pp. 199-210, 2014, doi: 10.14257/ijunesst.2014.7.2.19.

[28] B. Mallikarjuna, M. T. Student, K. H. Reddy, and O. Hemakesavulu, "Economic Load Dispatch Problem with Valve - Point Effect Using a Binary Bat Algorithm," ACEEE Int. J. Electr. Power Eng., vol. 4, no. 3, pp. 33-38, 2013.

[29] T. Jayabarathi, T. Raghunathan, B. R. Adarsh, and P. N. Suganthan, "Economic dispatch using hybrid grey wolf optimizer," Energy, vol. 111, pp. 630-641, 2016, doi: 10.1016/j.energy.2016.05.105.

[30] E. Sayedi, M. M. Farsangi, M. Barati, and K. Y. Lee, “A modified Shuffled frog leaping algorithm for nonconvex economic dispatch problem," IEEE Power Energy Soc. Gen. Meet., pp. 1-8, 2012, doi: 10.1109/PESGM.2012.6345586.

[31] A. Y. Abdelaziz, E. S. Ali, and S. M. Abd Elazim, "Implementation of flower pollination algorithm for solving economic load dispatch and combined economic emission dispatch problems in power systems," Energy, vol. 101, pp. 506-518, Apr. 2016, doi: 10.1016/j.energy.2016.02.041.
[32] T. Niknam, F. Golestaneh, and M. S. Sadeghi, "O-Multiobjective teaching-learning-based optimization for dynamic economic emission dispatch," IEEE Syst. J., vol. 6, no. 2, pp. 341-352, 2012, doi: 10.1109/JSYST.2012.2183276.

[33] W. Sa-Ngiamvibool, S. Pothiya, and I. Ngamroo, "Multiple tabu search algorithm for economic dispatch problem considering valve-point effects," Int. J. Electr. Power Energy Syst., vol. 33, no. 4, pp. 846-854, 2011, doi: 10.1016/j.ijepes.2010.11.011.

[34] L. Wang and L. P. Li, "An effective differential harmony search algorithm for the solving non-convex economic load dispatch problems," Int. J. Electr. Power Energy Syst., vol. 44, no. 1, pp. 832-843, 2013, doi: 10.1016/j.ijepes.2012.08.021.

[35] B. Mohammadi-ivatloo, A. Rabiee, A. Soroudi, and M. Ehsan, "Imperialist competitive algorithm for solving non-convex dynamic economic power dispatch," Energy, vol. 44, no. 1, pp. 228-240, Aug. 2012, doi: 10.1016/j.energy.2012.06.034.

[36] I. N. The, O. F. Power, and I. Committee, "Present practices in the economic operation of power systems," IEEE Trans. Power Appar. Syst., vol. PAS-90, no. 4, pp. 1768-1775, 1971, doi: 10.1109/TPAS.1971.293169.

[37] A. Lee, Fred; Breipohl, "Reserve constrained economic dispatch with prohibited zones," IEEE Trans. Power Syst., vol. 53, no. 9, pp. 1689-1699, 1993.

[38] M. Rodrigues Barbosa Dos Santos et al., "A Proposed Methodology Involving Progressive Bounded Constraints and Interior-Exterior Methods in Smoothed Economic/ Environmental Dispatch Problems," IEEE Lat. Am. Trans., vol. 15, no. 8, pp. 1422-1431, 2017, doi: 10.1109/TLA.2017.7994788.

[39] J. I. de Granda-Orive, A. Alonso-Arroyo, and F. RoigVázquez, "¿Qué base de datos debemos emplear para nuestros análisis bibliográficos? Web of Science versus SCOPUS," Arch. Bronconeumol., vol. 47, no. 4, p. 213, 2011, doi: 10.1016/j.arbres.2010.10.007.

[40] M. Romero, Manuel; Olite, Diego; Álvarez, “La contaminación del aire: su repercusión como problema de salud," Rev. Cubana Hig. Epidemiol., vol. 44, p. 14, 2006.

[41] M. A. Abido, "Environmental/Economic Power Dispatch Using Multiobjective Evolutionary Algorithms," IEEE Trans. Power Syst., vol. 18, no. 4, pp. 1529-1537, 2003, doi: 10.1109/TPWRS.2003.818693.

[42] M. A. Abido, "A novel multiobjective evolutionary algorithm for environmental/economic power dispatch," Electr. Power Syst. Res., vol. 65, no. 1, pp. 71-81, 2003, doi: 10.1016/S0378-7796(02)00221-3.

[43] M. A. Abido, "Multiobjective Evolutionary Algorithms for Electric Power Dispatch Problem," IEEE Trans. Evol. Comput., vol. 10, no. 3, pp. 315-329, 2006, doi: 10.1109/TEVC.2005.857073.

[44] S. Agrawal, B. K. Panigrahi, and M. K. Tiwari, "Multiobjective particle swarm algorithm with fuzzy clustering for electrical power dispatch," IEEE Trans. Evol. Comput., vol. 12, no. 5, pp. 529-541, 2008, doi: 10.1109/TEVC.2007.913121.

[45] Y. M. Atwa and E. F. El-Saadany, "Optimal allocation of ESS in distribution systems with a high penetration of wind energy," IEEE Trans. Power Syst., vol. 25, no. 4, pp. 1815-1822, 2010, doi: 10.1109/TPWRS.2010.2045663.

[46] M. Basu, "Economic Environmental Dispatch Using MultiObjective Differential Evolution," Appl. Soft Comput. J., vol. 11, no. 2, pp. 2845-2853, 2011, doi: 10.1016/j.asoc.2010.11.014. 
[47] W. Gu et al., "Modeling, planning and optimal energy management of combined cooling, heating and power microgrid: A review," Int. J. Electr. Power Energy Syst., vol. 54, no. 2014, pp. 26-37, 2014, doi: 10.1016/j.ijepes.2013.06.028.

[48] M. E. Khodayar, L. Wu, and M. Shahidehpour, "Hourly coordination of electric vehicle operation and volatile wind power generation in SCUC," IEEE Trans. Smart Grid, vol. 3, no. 3, pp. 1271-1279, 2012, doi: 10.1109/TSG.2012.2186642.

[49] A. A. Moghaddam, A. Seifi, T. Niknam, and M. R. Alizadeh Pahlavani, "Multi-objective operation management of a renewable MG (micro-grid) with back-up micro-turbine/ fuel cell/battery hybrid power source," Energy, vol. 36, no. 11, pp. 6490-6507, 2011, doi: 10.1016/j.energy.2011.09.017.

[50] L. Suganthi, S. Iniyan, and A. A. Samuel, "Applications of fuzzy logic in renewable energy systems - A review," Renew. Sustain. Energy Rev., vol. 48, pp. 585-607, 2015, doi: 10.1016/j.rser.2015.04.037.
[51] B. C. Ummels, M. Gibescu, E. Pelgrum, W. L. Kling, and A. J. Brand, "Impacts of wind power on thermal generation unit commitment and dispatch," IEEE Trans. Energy Convers., vol. 22, no. 1, pp. 44-51, 2007, doi: 10.1109/TEC.2006.889616.

[52] H. zhi Wang, G. qiang Li, G. bing Wang, J. chun Peng, H. Jiang, and Y. tao Liu, "Deep learning based ensemble approach for probabilistic wind power forecasting," Appl. Energy, vol. 188, pp. 56-70, 2017, doi: 10.1016/j.apenergy.2016.11.111.

[53] A. Zakariazadeh, S. Jadid, and P. Siano, "Multi-objective scheduling of electric vehicles in smart distribution system," Energy Convers. Manag., vol. 79, pp. 43-53, 2014, doi: 10.1016/j.enconman.2013.11.042. 